Research Article

\title{
Effect of Rejuvenated Binder on the Pavement Rutting Performance at Different Aging Levels
}

\author{
Hesham A. Ali $\mathbb{D}^{1},{ }^{1}$ Farshad Haddadi $\mathbb{D}^{1}{ }^{1}$ Mojtaba Mohammadafzali, ${ }^{2}$ Greg Sholar, ${ }^{3}$ \\ and Howard Moseley ${ }^{3}$ \\ ${ }^{1}$ Department of Civil and Environmental Engineering, Florida International University, 10555 West Flagler Street, \\ EC 3605, Miami, FL 33174, USA \\ ${ }^{2}$ Advanced Infrastructure Design, 1 Crossroads Drive, Hamilton, NJ08691, USA \\ ${ }^{3}$ Florida Department of Transportation, Department of Bituminous Material, Tallahassee, FL 32399, USA
}

Correspondence should be addressed to Hesham A. Ali; heaali@fiu.edu

Received 23 August 2019; Revised 1 February 2020; Accepted 13 February 2020; Published 25 August 2020

Academic Editor: Marco Cannas

Copyright (C) 2020 Hesham A. Ali et al. This is an open access article distributed under the Creative Commons Attribution License, which permits unrestricted use, distribution, and reproduction in any medium, provided the original work is properly cited.

\begin{abstract}
The use of Reclaimed Asphalt Pavement (RAP) in virgin mixtures is an effective means of promoting sustainability. The objective of this research was to investigate the effect of rejuvenated binder on the rutting performance and moisture susceptibility of high RAP content mixtures. All mixtures were tested at three aging levels, one short-term aging level, and two long-term aging levels. The performance of the specimens and the level of diffusion were measured and compared, and the correlation between binder test and rutting test results was evaluated. Based on the results, the degree of diffusion is not a determining factor in the performance of the mixture. However, the amount of the rejuvenator and Superpave performance grading (PG) of the rejuvenated binder is the most effective parameter. Results indicate that the short-term aged recycled mix exhibits a better rutting resistance than the virgin mix when its HTPG is $6^{\circ} \mathrm{C}$ higher than the virgin mix.
\end{abstract}

\section{Introduction}

The road to sustainability passes through the process of recycling a greater amount of asphalt pavement. The inclusion of additional RAP in new mixes has been a prominent method for promoting sustainability. However, there is no extensive research on using high percentage of RAP in new mixes. The amount of blending between the aged binder of the RAP and the new binder and recycling agents is still questionable and needs more investigations. Different methods have been utilized to measure the amount of the blending that all share the same idea; they assume that the aggregates are surrounded by the film of asphalt that has different characteristics throughout its thickness. The blending of the old and new binder is categorized into three different scenarios of no blending (black rock), complete blending, and partial blending. The incomplete blending of the new binder/rejuvenator with the aged binder causes a nonhomogeneous layer of the asphalt surrounding the aggregates, and this condition could affect the mixture's performances, such as rutting and fatigue cracking [1].

Coffey et al. [2] used mechanistic-empirical design principles to investigate the impact of the degree of blending on the performance of the RAP. Results showed that the degree of blending was $85 \%$ to $90 \%$ for three RAP specimens that were tested. The degree of blending did not affect the rutting performance significantly in this study. However, if a rejuvenator is applied, opening the road to traffic, before allowing sufficient time for the diffusion of the rejuvenator, may compromise the pavement stability and increase the amount of damage in road infrastructures because the outer layers are still too soft [3-6]. Besides, such methods have been used by researchers who study the rheology of concrete material and estimate the amount of concrete shrinkage especially due to the amount of the blending between cement and other cementitious material [7]. 
In a study conducted by Mohammadafzali [8] it was found that the proper rejuvenator has a critical pressure aging vessel (PAV) Time $\geq 50$ hours and $I_{h} \geq 0.9$. The proper rejuvenator helps the rejuvenated binder to age slower and facilitate the use of recycled binder with higher initial PG, not compromising the long-term performance. Critical PAV time is the time it takes the rejuvenated binder to go from high-temperature PG (HTPG) of $67^{\circ} \mathrm{C}$ to $95^{\circ} \mathrm{C}$. $I_{\mathrm{h}}$ is the homogeneity index-a measure of how well the rejuvenator diffuses with the binder.

\section{Objectives}

In this research, rutting performance and moisture susceptibility of the hot mix asphalt is evaluated to see the effect of rejuvenated RAP binder on the mixture's short and longterm performance. Based on the literature, two parameters of HTPG and the homogeneity index are assumed to be the effective parameters. Thus, different types and dosages of rejuvenators are used to get different $P G$ s and degree of homogeneities in the binder coating the aggregates.

\section{Methodology and Testing Procedures}

To determine the HTPG and homogeneity of binder coating the aggregates, the binder was extracted and then was tested by the dynamic shear rheometer (DSR) to determine the HTPG values [9]. Then the homogeneity index was calculated for each type of mixture as described in Section 3.1.

As the next step, the loaded wheel tester (LWT) was used to measure rutting performance and the moisture susceptibility of mixtures in accordance with AASHTO T 324 [10]. Finally, the correlation between LWT and binder test results was investigated.

3.1. Binder Extraction and Homogeneity Index. The binder was extracted using a centrifuge extractor and a rotary evaporator in accordance with ASTM D2172 and ASTM D5404, respectively $[11,12]$. To prepare specimens for binder extraction, the RAP and aggregates were heated to $165^{\circ} \mathrm{C}$ for $45 \pm 5$ minutes. Then, the rejuvenator or/and binder was added to the heated RAP or aggregate specimens and mixed for five minutes. The extraction specimen weight was $1100 \pm 20$ grams.

To do the extraction, the specimens were soaked in trichloroethylene at three stages: first: 1 minute, second: 3 minutes, and third for 45 minutes. Before the first extraction, aggregates are completely coated by a relatively thick layer of asphalt. The first extraction washes a large portion of the asphalt film away, leaving a thinner layer. After the second extraction, only a very thin layer of asphalt remains on the aggregates. The extracted liquid was then placed into a centrifuge with an 800 relative centrifugal force (RCF) for 30 minutes to make suspended fine aggregate sediment. The rotatory evaporator was used to separate the solvent from the binder. The binder extracted from the first, second, and third stages represents the outer, intermediate, and inner layers of the asphalt binder film coating the aggregates, respectively.
Finally, the binder extracted at each stage was tested by the DSR to determine the HTPG values at the outer, intermediate, and inner layers, according to AASHTO M320 criteria [13]. Homogeneity indices and stiffness gradient factors were calculated based on the HTPG of each layer.

Then, equation (1) is used to calculate the weighted average PG of the specimens adjusting for the individual layer mass:

$$
P G \text { ave }=a_{1} P G_{x 1}+a_{2} P G_{x 2}+a_{3} P G_{x 3} .
$$

in which,

$\mathrm{a}_{i}=\frac{\text { the mass of the binder recovered in stage of extraction }}{\text { mass of total recovered binder }}$.

where $P G_{x i}=$ the high-temperature $P G$ of the ith layer.

To quantify the homogeneity of the binder coating the aggregates, the homogeneity index $\left(I_{h}\right)$ (equation 3 ) is used [8]:

$$
I_{h}=1-\frac{\operatorname{Max} P G_{i}-\operatorname{Min} P G_{i}}{P G a v e},
$$

where $I_{h}$ is the degree of homogeneity of the binder coating the aggregates. Max $P G_{i}$ is the maximum $P G$ of the extracted layers, and Min $P G_{i}$ is the minimum $P G$ of the extracted layers.

An $I_{h}$ of 1 represents the most homogenous coating, and a smaller $I_{h}$ indicates less homogeneity. Based on the former research results, the $I_{h}$ value could vary between 0.75 and 0.99 , depending on the type of material and level of aging [14].

3.2. Loaded Wheel Tester (LWT). Four specimens (two specimens for each wheel) were tested to measure the rut depth at the $20,000^{\text {th }}$ cycle. Also, the stripping inflection point (SIP), which is a measure of the potential for moisture damage, was determined for all mixes. The rut depth and load cycle output data were initially fitted to a typical curve. The SIP, which is the inflection point of the fitted curve, was determined by finding the second derivative of the curve and equating it to zero [10].

\section{Material Preparation}

Two types of rejuvenators, one type of RAP, one virgin asphalt binder, and virgin aggregate, were used to prepare the specimens. Properties of these materials are explained in this section.

RAP material was obtained from a RAP stockpile in Miami Florida and tested to characterize the gradation of the material and the rheological characteristics of the binder. Theoretical maximum specific gravity $\left(\mathrm{G}_{\mathrm{mm}}\right)$ of the RAP material is 2.415 and contains $7.24 \%$ binder with high PG of $89.53^{\circ} \mathrm{C}$. Virgin aggregate was obtained from a local asphalt plant. The gradation of RAP, screening, and virgin aggregate is presented in Table 1.

Virgin asphalt binder was obtained from a local asphalt producer (General Asphalt). HTPG of the recovered binder 
TABLE 1: Gradation of the RAP, screening, and virgin aggregate.

\begin{tabular}{lcccccccccc}
\hline Sieve size & $19 \mathrm{~mm}$ & $12.5 \mathrm{~mm}$ & $9.5 \mathrm{~mm}$ & No. 4 & No. 8 & No. 16 & No. 30 & No. 50 & No. 100 & No. 200 \\
\hline Passing\% (RAP) & 100.00 & 99.42 & 93.52 & 67.26 & 52.19 & 39.94 & 32.94 & 22.11 & 7.43 & 4.83 \\
Passing\% (screening) & 100 & 100 & 100 & 100 & 92 & 72 & 56 & 41 & 13 & 2.3 \\
Passing\% (virgin aggregate) & 100 & 99.67 & 93.24 & 66.87 & 52.34 & 40.16 & 32.18 & 21.54 & 7.09 & 3.47 \\
\hline
\end{tabular}

was determined using the DSR, per AASHTO T315 [9]. The high-temperature PG was $74.2^{\circ} \mathrm{C}$.

Two types of rejuvenators were used in this study: named as RA1 and RA2. RA1 is a dark yellow heavy paraffinic oil with a high aromatic content that provides good softening power. The rejuvenator contains no asphaltene. RA2 is an oil extract that contains about half aromatic and half naphthenic molecules to maintain compatibility between the asphalt and the rejuvenator oil.

The stage extraction process (Section 5.1) was performed on the rejuvenated samples after five days of oven aging at $85^{\circ} \mathrm{C}$. Table 2 shows the results of the stage extractions and resulting homogeneity indices $\left(I_{h}\right)$. RA2 had a smaller $I_{h}$ of 0.91 compared to RA1 with $I_{h}$ of 0.95 . This indicates that RA2 has relatively inferior diffusion characteristics in comparison to RA1 [14]. Both rejuvenators have critical PAV time of more than 50 hours.

\section{Specimen Preparation}

5.1. Preparation of the Specimens. The rejuvenated specimens were prepared by mixing the RAP with designed quantities of rejuvenator and screening sand. The loose RAP was heated at $165^{\circ} \mathrm{C}$ for one hour. Next, a rejuvenator was introduced, and the specimen was mixed in a bowl mixer for five minutes. The control specimens were produced by mixing heated virgin aggregate with $6.4 \%$ of virgin asphalt binder. The process for fabricating the specimens by a gyratory compactor included heating the mixtures to $165^{\circ} \mathrm{C}$ for one hour. This exposure was considered short-term aging that all specimens were exposed to. LWT test was conducted on specimens with three aging conditions; one specimen from each type of mixtures was tested without any long-term aging. The second and third specimens underwent simulated long-term aging by being heated at $85^{\circ} \mathrm{C}$ for five days and ten days, respectively. The LWT test required air void values of $7 \pm 0.5$ percent. All specimens had a height of $60 \mathrm{~mm}$ and a diameter of $150 \mathrm{~mm}$.

5.2. Specimen Design. Table 3 shows the general composition of the specimens and the aging and the target PG and dosage of rejuvenators for each specimen. In this research the PG of specimens $\mathrm{R} 3$ and $\mathrm{R} 4$ was assumed to be $6^{\circ} \mathrm{C}$ higher than that of the control to test if a recycled mixture can have a similar long-term performance to that of a virgin mixture, with a $6^{\circ} \mathrm{C}$ lower PG.

5.3. Rejuvenated Specimens. After making several trial specimens, it was concluded that adding screenings with a quantity almost equal to that of the rejuvenator leads to consistent air void values (between three to four percent).
The gradation of the screenings is presented in Table 1. It is well-graded limestone sand with a low passing \#200 content. The air void values were determined after compacting the specimens by 50 gyrations using a gyratory compactor. The amount of rejuvenator added to each specimen was determined using the softening curves presented in Figure 1. Table 4 shows the composition of the specimens, and the $\mathrm{G}_{\mathrm{mm}}$, and air void values.

5.4. Control (Virgin Specimens). The control specimens consisted of virgin asphalt and virgin aggregate. The aggregates were sieved to individual sizes and were then composed to obtain almost the same gradations as the average gradation for the RAP (Table 1). Table 5 shows the composition and volumetric properties of the control specimens, and Table 1 shows the gradation of the virgin aggregate.

\section{Experimental Results and Interpretation}

6.1. DSR Test. The average $I_{h}$ of the three aging levels for each specimen type is presented in Table 6 . The lowest $I_{h}$ belongs to the virgin binder with 0.87 and the highest belongs to R1 and R3, which contain rejuvenator RA1. The $I_{h}$ of 0.87 means that for the control specimens, the outer layer of the virgin binder ages much faster than the rejuvenated binder in R1, R2, R3, and R4. On the other hand, the $I_{h}$ of the 0.93 means that the binder in the R1 and R2 specimens, containing RA1 rejuvenator, ages more consistently along with the coating layer in comparison to other specimens.

6.2. Loaded Wheel Testing. Figure 2 presents rut depth measurements at 20,000 passes for mixes evaluated. Table 7 shows the average rut depth at 20,000 passes, along with the coefficient of variation (COV) and statistical ranking of mixes evaluated. The average COV was $18.6 \%$, with a range of $5 \%-30 \%$. Table 8 summarizes the average stripping inflection point (SIP), along with the statistical ranking of mixes evaluated. The average SIP COV was $12.1 \%$, with a range of $6 \%-22 \%$.

The short-term aged virgin mix and recycled mixes with high rejuvenator dosage rates (RAP $+15.7 \% \quad \mathrm{RA} 1$ and RAP $+11.3 \%$ RA2) exhibited higher rut depths than similar mixes that were long-term aged at five and ten days. However, the evaluated aging levels did not seem to affect the rut depths for recycled mixes at lower rejuvenator dosage levels (RAP+7.9\% RA1 and RAP + 6.5\% RA2). This indicates that the higher rejuvenator dosage was effective in softening the recycled binder and thereby increases rutting potential. This observation is consistent with the binder HTPG presented in Table 2. The softening effect is short-term, and the 
TABLE 2: Stage extractions for rejuvenator selection process.

\begin{tabular}{|c|c|c|c|c|c|c|c|c|}
\hline \multirow{2}{*}{ Rej. } & \multirow{2}{*}{ Extraction no. } & \multicolumn{2}{|c|}{ Recovered binder } & \multirow{2}{*}{$P G \mathrm{i}$} & \multirow{2}{*}{$P G_{\text {ave }}$} & \multirow{2}{*}{$P G i-P G_{\text {ave }}$} & \multirow{2}{*}{$\frac{P G i}{P G a v e}$} & \multirow{2}{*}{$I_{h}$} \\
\hline & & (Grams) & (Proportion) & & & & & \\
\hline \multirow{3}{*}{ RA1 } & 1 & 32.72 & 0.54 & 78.19 & \multirow{3}{*}{78.34} & -0.15 & 1.00 & \multirow{3}{*}{0.95} \\
\hline & 2 & 15.50 & 0.26 & 80.39 & & 2.05 & 1.03 & \\
\hline & 3 & 12.32 & 0.20 & 76.16 & & -2.18 & 0.97 & \\
\hline \multirow{3}{*}{ RA2 } & 1 & 33.18 & 0.57 & 81.91 & \multirow{3}{*}{78.87} & 3.04 & 1.04 & \multirow{3}{*}{0.91} \\
\hline & 2 & 14.20 & 0.24 & 74.83 & & -4.04 & 0.95 & \\
\hline & 3 & 11.02 & 0.19 & 74.93 & & -3.95 & 0.95 & \\
\hline
\end{tabular}

TABle 3: Factorial design of the experiment.

\begin{tabular}{|c|c|c|c|c|c|}
\hline \multirow{3}{*}{ Specimen name } & \multirow{3}{*}{ Composition } & \multirow{3}{*}{ High-temperature PG $\left({ }^{\circ} \mathrm{C}\right)$} & \multicolumn{3}{|c|}{ Cell number } \\
\hline & & & \multicolumn{3}{|c|}{ Aging } \\
\hline & & & No additional aging & 5 Days at $85^{\circ} \mathrm{C}$ & 10 Days at $85^{\circ} \mathrm{C}$ \\
\hline Control & RAP aggregate + virgin binder & $74.2 \pm 1^{\circ} \mathrm{C}$ & 1 & 2 & 3 \\
\hline $\mathrm{R} 1$ & RAP $+15.7 \%$ RA 1 & $74.2 \pm 1^{\circ} \mathrm{C}$ & 4 & 5 & 6 \\
\hline $\mathrm{R} 2$ & $\mathrm{RAP}+11.3 \% \mathrm{RA} 2$ & $74.2 \pm 1^{\circ} \mathrm{C}$ & 7 & 8 & 9 \\
\hline R3 & $\mathrm{RAP}+7.9 \% \mathrm{RA} 1$ & $80.2 \pm 1^{\circ} \mathrm{C}$ & 10 & 11 & 12 \\
\hline $\mathrm{R} 4$ & $\mathrm{RAP}+6.5 \% \mathrm{RA} 2$ & $80.2 \pm 1^{\circ} \mathrm{C}$ & 13 & 14 & 15 \\
\hline
\end{tabular}

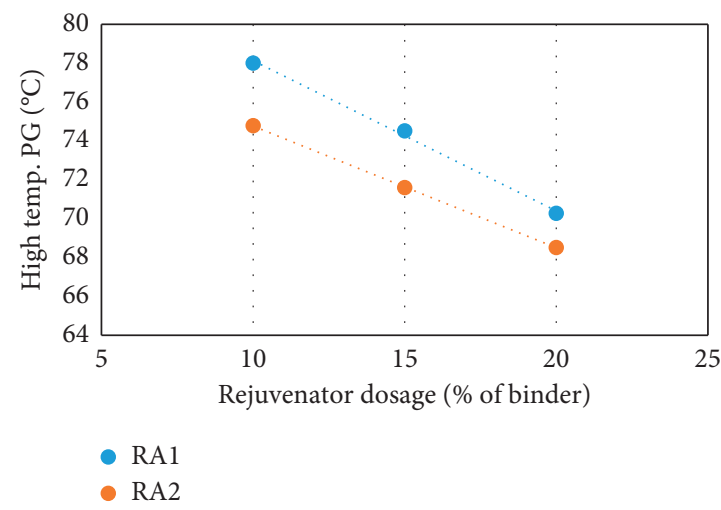

FIGURE 1: Softening curves of rejuvenators.

TABLE 4: Composition and volumetric properties of the rejuvenated specimens.

\begin{tabular}{|c|c|c|c|c|c|}
\hline \multirow{2}{*}{ Specimen } & \multirow{2}{*}{ Rejuvenator } & \multicolumn{2}{|c|}{ Rejuvenator content } & \multirow{2}{*}{ Screenings content (\%) } & \multirow{2}{*}{ Density $\left(\% \mathrm{G}_{\mathrm{mm}}\right)$} \\
\hline & & By binder weight (\%) & By mix weigh (\%) & & \\
\hline R1 & & 15.7 & 1.14 & 1.14 & 96.52 \\
\hline R3 & RAI & 7.9 & 0.87 & 0.87 & 96.98 \\
\hline R2 & & 11.3 & 0.57 & 0.57 & 96.94 \\
\hline $\mathrm{R} 4$ & RA2 & 6.5 & 0.29 & 0.20 & 96.31 \\
\hline
\end{tabular}

TABle 5: Composition and volumetric properties of the control specimens.

\begin{tabular}{lcccc}
\hline Specimen & Passing \#200 (\%) & Binder content (\%) & $\mathrm{G}_{\mathrm{mm}}$ & $\mathrm{G}_{\mathrm{mb}} \%$ \\
\hline Control & 3.5 & 6.4 & 2.352 & 96.64
\end{tabular}

$\mathrm{G}_{\mathrm{mm}}$ : theoretical maximum specific gravity; $\mathrm{G}_{\mathrm{mb}}$ : specimen's bulk specific gravity; $G_{m b} \%: G_{m m}$ to $G_{m b}$ ratio.

mix rutting performance improves with aging. It is noted that the RAP $+7.9 \%$ RA1 mix which aged for ten days was
TABLE 6: Average $I_{h}$ of the three aging levels of each specimen type.

\begin{tabular}{lccccc}
\hline Specimen name & Control & R1 & R2 & R3 & R4 \\
\hline$I_{h}$ & 0.87 & 0.93 & 0.92 & 0.93 & 0.89 \\
\hline
\end{tabular}

damaged and was not tested. This is shown in Tables 7 and 8 as Not Measured (NM).

The effects of aging on moisture susceptibility are reported as the stripping inflection point (Table 8). An increased aging level improved moisture damage resistance for 


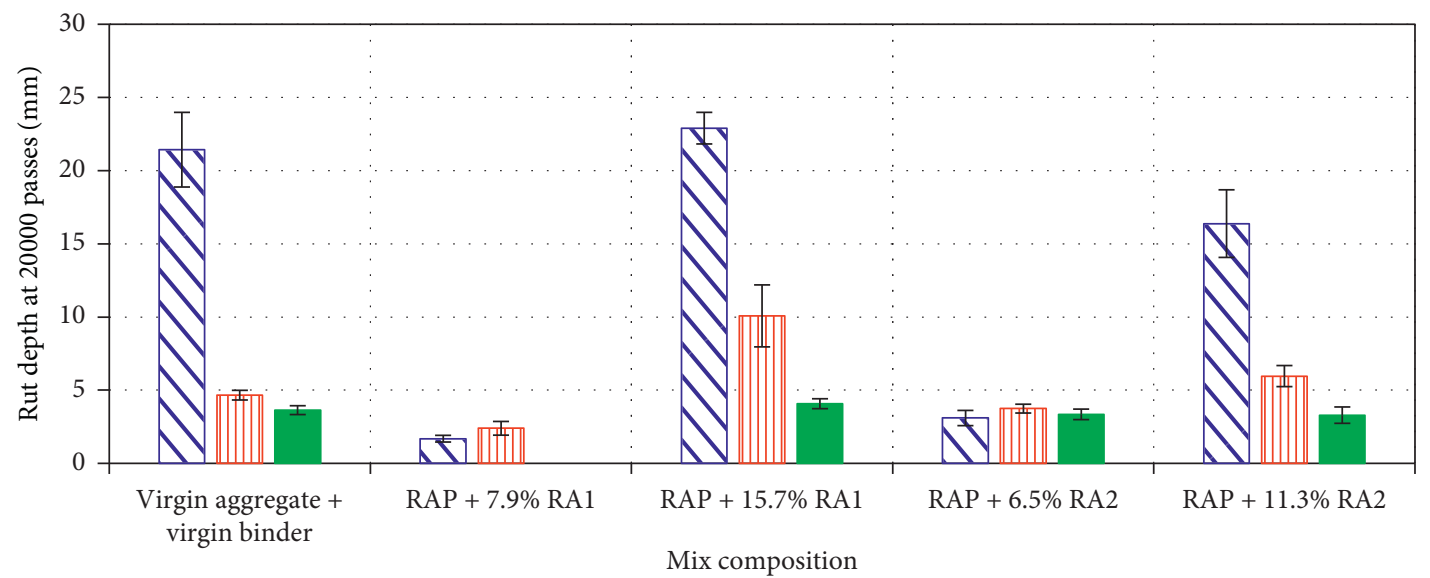

$\mathbb{\square}$ 1-hr aging at $165^{\circ} \mathrm{C}$

晸 5 -day aging at $85^{\circ} \mathrm{C}$

Figure 2: LWT test results, $50^{\circ} \mathrm{C}$ wet.

TABLE 7: LWT rut depth test results, $50^{\circ} \mathrm{C}$ wet.

\begin{tabular}{|c|c|c|c|c|}
\hline \multirow{2}{*}{ Mix aging level } & \multirow{2}{*}{ Mix composition } & \multicolumn{3}{|c|}{ Rut depth at 20000 passes $(\mathrm{mm})$} \\
\hline & & Average & Std. Dev. & Groupings \\
\hline \multirow{5}{*}{ 1-hr aging at $165^{\circ} \mathrm{C}$} & Virgin aggregate + Virgin binder & 21.4 & 3.6 & $\mathrm{C}$ \\
\hline & RAP + 7.9\% RA1 & 1.7 & 0.3 & $\mathrm{~A}$ \\
\hline & $\mathrm{RAP}+15.7 \% \mathrm{RA} 1$ & 22.9 & 1.2 & $\mathrm{C}$ \\
\hline & $\mathrm{RAP}+6.5 \% \mathrm{RA} 2$ & 3.1 & 0.7 & A \\
\hline & $\mathrm{RAP}+11.3 \% \mathrm{RA} 2$ & 16.4 & 3.3 & $\mathrm{~B}$ \\
\hline \multirow{5}{*}{5 -day aging at $85^{\circ} \mathrm{C}$} & Virgin aggregate + virgin binder & 4.7 & 0.5 & $\mathrm{~B} / \mathrm{C}$ \\
\hline & RAP + 7.9\% RA1 & 2.4 & 0.7 & $\mathrm{~A}$ \\
\hline & $\mathrm{RAP}+15.7 \% \mathrm{RA} 1$ & 10.1 & 3.0 & $\mathrm{D}$ \\
\hline & $\mathrm{RAP}+6.5 \% \mathrm{RA} 2$ & 3.7 & 0.4 & $\mathrm{~A} / \mathrm{B}$ \\
\hline & $\mathrm{RAP}+11.3 \% \mathrm{RA} 2$ & 6.0 & 1.0 & $\mathrm{C}$ \\
\hline \multirow{5}{*}{10 -day aging at $85^{\circ} \mathrm{C}$} & Virgin aggregate + virgin binder & 3.6 & 0.4 & $\mathrm{~A} / \mathrm{B}$ \\
\hline & RAP + 7.9\% RA1 & NM & NA & NA \\
\hline & $\mathrm{RAP}+15.7 \% \mathrm{RA} 1$ & 4.1 & 0.5 & $\mathrm{~B}$ \\
\hline & $\mathrm{RAP}+.5 \% \mathrm{RA} 2$ & 3.3 & 0.5 & A \\
\hline & $\mathrm{RAP}+11.3 \%$ RA 2 & 3.3 & 0.8 & $\mathrm{~A}$ \\
\hline
\end{tabular}

NA: not applicable; NM: not measured as the specimen was damaged; COV: coefficient of variation. Statistical groupings were based on Tukey's analysis at a $95 \%$ confidence level.

TABLE 8: LWT stripping inflection point results, $50^{\circ} \mathrm{C}$ wet.

\begin{tabular}{|c|c|c|c|c|}
\hline \multirow{2}{*}{ Mix composition } & \multirow{2}{*}{ Aging level } & \multicolumn{3}{|c|}{ Stripping inflection point (number of passes) } \\
\hline & & Average & $\operatorname{COV}(\%)$ & Groupings \\
\hline \multirow{3}{*}{ Virgin aggregate + virgin binder } & 1 -hr aging at $165^{\circ} \mathrm{C}$ & 6225 & 10 & $\mathrm{~B}$ \\
\hline & 5 -day aging at $85^{\circ} \mathrm{C}$ & $20000^{* *}$ & NA & $\mathrm{A}$ \\
\hline & 10 -day aging at $85^{\circ} \mathrm{C}$ & $20000^{* *}$ & NA & $\mathrm{A}$ \\
\hline \multirow{3}{*}{$\mathrm{RAP}+7.9 \% \mathrm{RA} 1$} & 1-hr aging at $165^{\circ} \mathrm{C}$ & $20000^{* *}$ & NA & $\mathrm{A}$ \\
\hline & 5 -day aging at $85^{\circ} \mathrm{C}$ & $20000^{* *}$ & NA & A \\
\hline & 10 -day aging at $85^{\circ} \mathrm{C}$ & NM & NA & NA \\
\hline \multirow{3}{*}{$\mathrm{RAP}+15.7 \% \mathrm{RA} 1$} & 1-hr aging at $165^{\circ} \mathrm{C}$ & 5683 & 12 & $\mathrm{C}$ \\
\hline & 5 -day aging at $85^{\circ} \mathrm{C}$ & 14344 & 22 & $\mathrm{~B}$ \\
\hline & 10-day aging at $85^{\circ} \mathrm{C}$ & $20000^{* *}$ & NA & A \\
\hline \multirow{3}{*}{$\mathrm{RAP}+6.5 \% \mathrm{RA} 2$} & 1-hr aging at $165^{\circ} \mathrm{C}$ & $20000^{* *}$ & NA & $\mathrm{A}$ \\
\hline & 5 -day aging at $85^{\circ} \mathrm{C}$ & $20000^{* *}$ & NA & $\mathrm{A}$ \\
\hline & 10 -day aging at $85^{\circ} \mathrm{C}$ & $20000^{* *}$ & NA & $\mathrm{A}$ \\
\hline \multirow{3}{*}{$\mathrm{RAP}+11.3 \% \mathrm{RA} 2$} & 1-hr aging at $165^{\circ} \mathrm{C}$ & 11238 & 6 & $\mathrm{C}$ \\
\hline & 5 -day aging at $85^{\circ} \mathrm{C}$ & 15790 & 9 & $\mathrm{~B}$ \\
\hline & 10-day aging at $85^{\circ} \mathrm{C}$ & $20000^{* *}$ & NA & $\mathrm{A}$ \\
\hline
\end{tabular}

NA: not applicable (mixes did not strip after 20000 passes); NM: not measured as the specimen was damaged; COV: coefficient of variation. Statistical groupings were based on Tukey's analysis at a 95\% confidence level. 
TABLE 9: Linear correlation between rut depth and independent variables.

\begin{tabular}{lccr}
\hline & & & Linear regression $(n=15)$ \\
& & R-squared & $p$-value \\
\hline 1 & $\mathrm{PG}_{\text {ave }}$ & 0.464 & 0.005 \\
2 & $X_{1}$ & 0.443 & 0.007 \\
3 & $X_{2}$ & 0.381 & 0.014 \\
4 & $X_{3}$ & 0.359 & 0.018 \\
5 & $I_{\mathrm{h}}$ & 0.029 & 0.544 \\
\hline
\end{tabular}

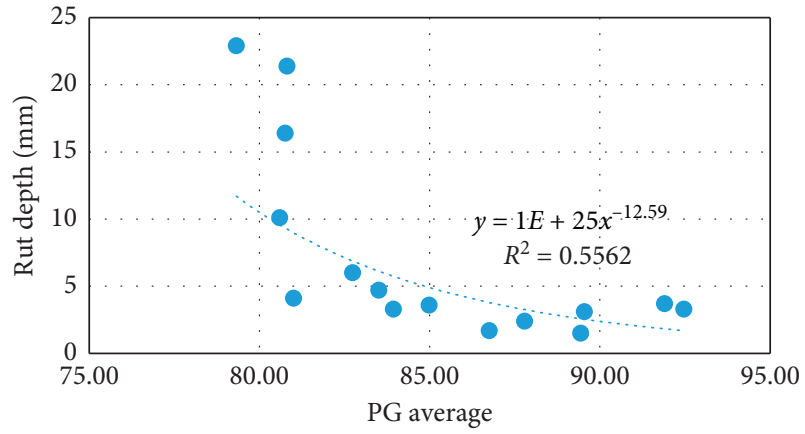

FIgURE 3: Correlation between $\mathrm{PG}_{\mathrm{ave}}$ and rut depth $(n=15)$.

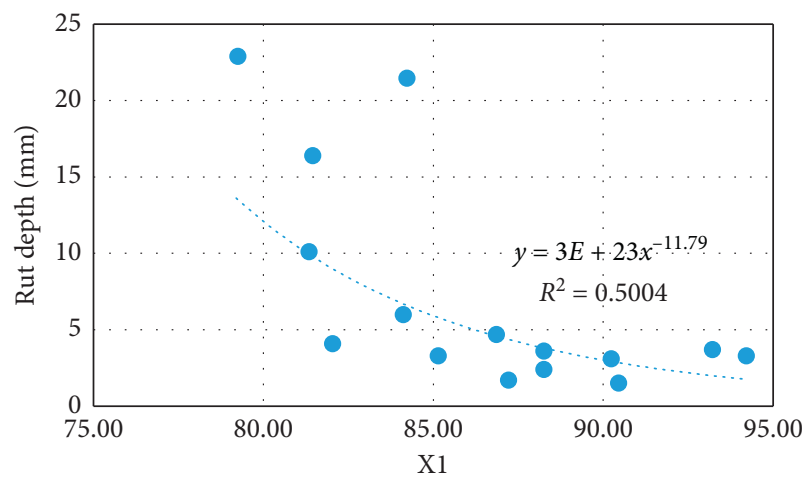

Figure 4: Correlation between X1 and rut depth $(n=15)$.

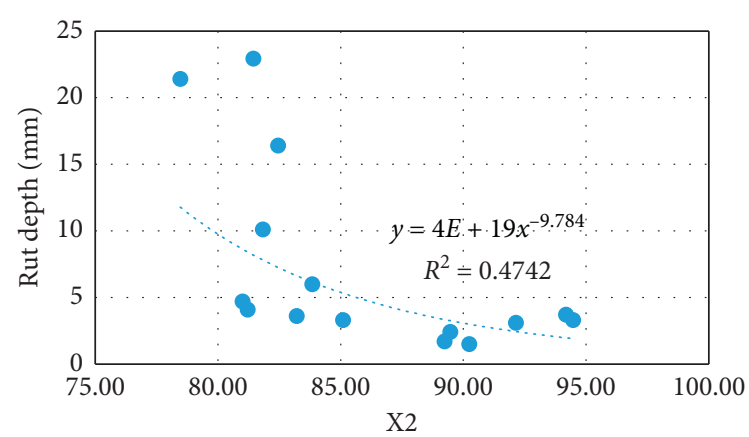

FIgURE 5: Correlation between X2 and rut depth $(n=15)$.

recycled mixes with a higher rejuvenator dosage rate. Table 8 shows that for RAP specimens with a $6^{\circ} \mathrm{C}$ higher HTPG than virgin mix and specimens $2,3,9$, and 15, no stripping inflection was observed within 20,000 passes.

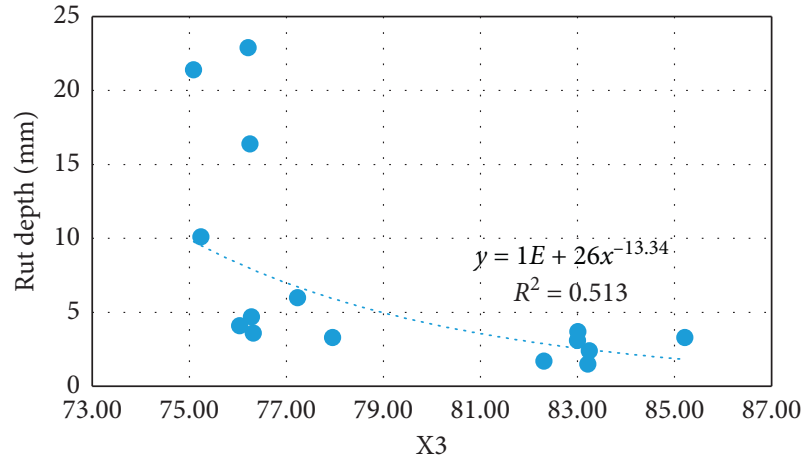

Figure 6: Correlation between X3 and rut depth $(n=15)$.

TABLE 10: Nonlinear correlation between rut depth and independent variables.

\begin{tabular}{ccccc}
\hline & & R-squared & $\mathrm{a}$ & $\mathrm{b}$ \\
\hline 1 & $\mathrm{PG}_{\text {ave }}$ & 0.556 & $10^{25}$ & -12.59 \\
2 & $X_{1}$ & 0.500 & $3 \times 10^{23}$ & -11.79 \\
3 & $X_{2}$ & 0.474 & $4 \times 10^{19}$ & -9.784 \\
4 & $X_{3}$ & 0.513 & $10^{26}$ & -13.34 \\
\hline
\end{tabular}

\section{Correlations}

Rutting results for all 15 specimens are correlated with characteristics of the extracted binder, and the results are summarized in Table 9. The R-squared and the significance level of the correlations are calculated using SPSS software. The R-squared measures the strength of the relationship between the model and the dependent variable on a convenient $0-1$ scale. The $p$-value for each term tests the null hypothesis that the coefficient is equal to zero (no effect). A low $p$-value $(<0.05)$ indicates that we can reject the null hypothesis. In other words, a predictor that has a low $p$-value is likely to be a meaningful addition to the model because changes in the predictor's value are related to changes in the response variable.

Layers $X_{1}, X_{2}$, and $X_{3}$ represent the stiffness of the outer, middle, and inner "layers" of the binder coating around the aggregates. As shown in Table 9, the PGave and X1 have the most significant correlations with the rut depth, with 0.005 and $0.007 p$-values, respectively. Stiffness of the second and third layers also somewhat correlated with rutting, which is consistent with expectation. The trend curve shown in Figures 3 to 6 demonstrates the inverse relationship between variables 1 to 4 with the rut depth. This means that as the 
HTPG increases, the rutting susceptibility of the mix decreases.

$I_{h}$ did not show any significant correlations with rutting. The power function $\left(y=a * \mathrm{X}^{\mathrm{b}}\right)$ was also fitted to the data, which results in a higher R-squared. Parameters $a, b$, and $\mathrm{R}$-squared for power function regression are presented in Table 10.

\section{Conclusion}

In this study, the rutting test was conducted on rejuvenated RAP specimens and compared to virgin asphalt concrete specimens, and the results were correlated with rheological characteristics of binder extracted from binder layers surrounding the aggregates. After analyzing the results, the following conclusions were made:

(i) The short-term aged recycled mix in this study exhibited better rutting resistance than the virgin mix when its HTPG was $6^{\circ} \mathrm{C}$ higher than the virgin mix. When it had the same HTPG, the rutting resistance was similar. Long-term aged (10-day) recycled mixes recorded rutting and moisture resistance similar to the virgin mixes.

(ii) The virgin (control) mix had an average rut depth of $10 \mathrm{~mm}$. The average rut depth for recycled mixes with HTPG of the same as the virgin mix was $10.46 \mathrm{~mm}$ and with recycled mixes, with $6^{\circ} \mathrm{C}$ higher HTPG was $2.84 \mathrm{~mm}$. This indicates that the overall rutting performance of the control mix was almost the same as the recycled mix when the HTPG is the same. Also, this indicates that the recycled mix had an overall better rutting resistance than the virgin mix when its HTPG was $6^{\circ} \mathrm{C}$ higher than the virgin mix.

(iii) Generally, short-term aged (1 hour) recycled mixes with a higher HTPG $\left(6^{\circ} \mathrm{C}\right.$ higher than that of virgin binder) exhibited less rutting and higher moisture resistance than short-term aged virgin mixes. The test results for 5-day aged mixes were mixed for rutting and moisture resistance. Long-term aged (10-day) recycled mixes presented similar rutting and moisture resistance as the virgin mixes.

(iv) Both rejuvenators used in this study had a PAV critical time of more than 50 hours but different homogeneity index. Although RA1 had a higher homogeneity index than RA2, there is no significant difference between the rutting performance of the mixtures fabricated by RA1 and RA2.

\section{Data Availability}

The data used to support the findings of this study are available from the corresponding author upon request and permission from the funder and material suppliers.

\section{Conflicts of Interest}

The authors declare that there are no conflicts of interest regarding the publication of this paper.

\section{Acknowledgments}

This research was funded by the Florida Department of Transportation. The authors thank LSU for testing support. The authors express appreciation to General Asphalt and rejuvenator suppliers for material supply.

\section{References}

[1] B. Huang, G. Li, D. Vukosavljevic, X. Shu, and B. K. Egan, "Laboratory investigation of mixing hot-mix asphalt with reclaimed asphalt pavement," Transportation Research Record: Journal of the Transportation Research Board, vol. 1929, no. 1, pp. 37-45, 2005.

[2] S. Coffey, E. DuBois, Y. Mehta, A. Nolan, and C. Purdy, "Determining the impact of degree of blending and quality of reclaimed asphalt pavement on predicted pavement performance using pavement ME design," Construction and Building Materials, vol. 48, pp. 473-478, 2013.

[3] M. Zaumanis, R. B. Mallick, L. Poulikakos, and R. Frank, "Influence of six rejuvenators on the performance properties of reclaimed asphalt pavement (RAP) binder and 100\% recycled asphalt mixtures," Construction and Building Materials, vol. 71, pp. 538-550, 2014.

[4] M. Abedin, S. Farhangdoust, and A. Mehrabi, "Fracture detection in steel girder bridges using self-powered wireless sensors," in Proceedings of the in Risk-Based Bridge Engineering: Proceedings of the 10th New York City Bridge ConferenceBridge Engineering Association, New York, NY, USA, August, 2019.

[5] D. Garber and E. Shahrokhinasab, Performance Comparison of In-Service Full-Depth Precast Concrete Deck Panels to Cast-In-Place Decks, Accelerated Bridge Construction University Transportation Center (ABC-UTC), Miami, FL, USA, 2019.

[6] Y. Xu, S. Xu, and J. Ji, "Measurement method of blending status between virgin and aged binder in recycled asphalt mixtures-a literature review," In Challenges and Advances in Sustainable Transportation Systems, pp. 311-318, 2014.

[7] P. Ghoddousi, A. M. Abbasi, E. Shahrokhinasab, and M. Abedin, "Prediction of plastic shrinkage cracking of selfcompacting concrete," Advances in Civil Engineering, vol. 2019, Article ID 1296248, 7 pages, 2019.

[8] M. Mohammadafzali, Evaluation of Durability and Homogeneity of Rejuvenated Asphalt Binders, Florida International University, Miami, FL, USA, 2017, https://digitalcommons. fiu.edu/etd/3167/.

[9] AASHTO, Standard Method of Test for Determining the Rheological Properties of Asphalt Binder Using a Dynamic Shear Rheometer (DSR), AASHTO T315, Washington, DC, USA, 2009b.

[10] T. 324 AASHTO, S Standard Method of Test for Hamburg Wheel-Track Testing of Compacted Asphalt Mixtures, American Association of State Highway and Transportation Officials, Washington, DC, USA, 2017.

[11] ASTM-D2172, Standard Test Methods for Quantitative Extraction of Asphalt Binder from Asphalt Mixtures, ASTM International, West Conshohocken, PA, USA, 2017.

[12] ASTM-D5404, Standard Practice for Recovery of Asphalt from Solution Using the Rotary Evaporator, ASTM International, West Conshohocken, PA, USA, 2017. 
[13] M. 320 AASHTO, Standard Specification for PerformanceGraded Asphalt Binder, American Association of State Highway and Transportation Officials, Washington, DC, USA, 2017.

[14] H. Ali, L. Mohammad, M. Mohammadafzali, F. Haddadi, and M. Akentuna, Evaluation of the Effect of Homogeneity of the Asphalt Binder on Performance of a Recycled Mix, Florida Department of Transportation, Tallahassee, FL, USA, 2019. 